\title{
A Importância da Referenciação Precoce na Falência Hepática Aguda Pediátrica
}

\author{
The Importance of Early Referral in Pediatric Acute Liver Failure
}



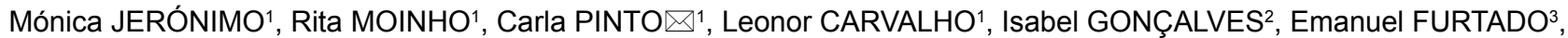 \\ José FARELA NEVES ${ }^{1}$ \\ Acta Med Port 2015 Sep-Oct;28(5):559-566
}

\section{RESUMO}

Introdução: A falência hepática aguda é uma doença rara associada a elevada morbilidade e mortalidade apesar do aumento da sobrevida devido ao transplante hepático. Em 2008, decorreu em Portugal uma reunião sobre esta patologia em pediatria, resultando num consenso de atuação que salientou a importância da abordagem multidisciplinar e referenciação precoce para um centro de transplantação hepática.

Objetivos: Caracterizar as admissões por falência hepática aguda no Serviço de Cuidados Intensivos Pediátricos do centro português com transplante hepático pediátrico. Comparar resultados antes (A) e depois de 2008 (B).

Material e Métodos: Estudo observacional retrospetivo de 20 anos (1994-2014). Critérios de inclusão: idade < 18 anos e falência hepática aguda (INR $\geq 2$ sem resposta à vitamina $\mathrm{K}$ e necrose hepatocelular). Excluíram-se as crianças com doença hepática crónica. Resultados: Incluíram-se 50 crianças com idade mediana de 24,5 meses. A causa mais comum de falência hepática aguda abaixo dos 2 anos foi metabólica (34,6\%) e acima infeciosa (29,2\%). Foram submetidos a transplante hepático $46 \%$, tendo sobrevivido $78 \%$. A mortalidade global foi 34\%. A mediana do tempo de referenciação foi 7 dias no período $A(n=35)$ e 2 no $B(n=15 ; p=0,006)$. A mediana do risco de mortalidade prevista pelo PRISM foi $14,7 \%$ no período A e $6,5 \%$ no B $(p=0,019)$. A mortalidade foi $37 \%$ vs $26 \%$ no período $\mathrm{A}$ e $\mathrm{B}$ respetivamente $(p=0,474)$.

Discussão e Conclusões: A mortalidade global foi sobreponível à de outros centros europeus, sendo o transplante hepático a opção terapêutica mais eficaz. Após 2008 o tempo de referenciação e a gravidade dos casos na admissão reduziram, ainda sem tradução significativa na mortalidade.

Palavras-chave: Criança; Falência Hepática Aguda; Referenciação; Transplante de Fígado.

\section{ABSTRACT}

Introduction: Acute liver failure is a rare disorder associated to high morbidity and mortality despite survival improvement through liver transplantation. The importance of a multidisciplinary approach and early referral to a pediatric liver transplantation center were important conclusions of a national meeting in 2008, from which resulted an actuation consensus.

Objectives: To characterize acute liver failure admissions in a Pediatric Intensive Care Unit of the portuguese pediatric liver transplantation center. To compare results before (A) and after (B) 2008.

Material and Methods: Observational, retrospective study during a 20 year period (1994-2014). Inclusion criteria: age < 18 years old and acute liver failure (INR $\geq 2$ without vitamin $\mathrm{K}$ response and hepatocellular necrosis). Children with previous liver disease were excluded.

Results: Fifty children were included, with median age of 24.5 months. The most common etiology under 2 years old was metabolic $(34.6 \%)$ and above that age was infectious (29.2\%). Forty six percent were submitted to liver transplantation and $78 \%$ of them survived. Overall mortality was $34 \%$. Median referral time was 7 days in period $A(n=35)$ and 2 days in period $B(n=15 ; p=0.006)$. Pediatric risk of mortality's median was 14.7 in period A and 6.5 in B $(p=0.019)$. Mortality was $37 \%$ vs $26 \%$ in periods A and $B$, respectively $(p=0.474)$.

Discussion and Conclusions: Overall mortality was similar to the observed in other European centers. Liver transplantation is in fact the most effective therapeutic option. After 2008, there was a reduction in referral time and cases severity on admission; however, mortality has not reduced so far.

Keywords: Child; Liver Failure, Acute; Liver Transplantation; Referral and Consultation.

\section{INTRODUÇÃO}

A falência hepática aguda (FHA) é uma patologia rara em idade pediátrica, definida por necrose hepatocelular grave e envolvimento multissistémico, associando-se a elevada morbilidade e mortalidade. ${ }^{1-5}$

Segundo o grupo de estudo Pediatric Acute Liver Failure (PALF), a FHA é uma entidade patológica, em crianças sem doença hepática crónica, com evidência bioquímica de lesão hepática aguda e coagulopatia (tempo de protrombi- na $\geq 15$ segundos ou INR $\geq 1,5$ ) não corrigida com vitamina $\mathrm{K}$, na presença de encefalopatia hepática clínica ou, independentemente da ocorrência de encefalopatia, tempo de protrombina $\geq 20$ segundos ou INR $\geq 2,0$. 2,4-6

A FHA tem diversas etiologias que devem ser ativamente pesquisadas pois permitem orientar a terapêutica, constituindo um importante fator de prognóstico. ${ }^{1,5,6}$ As causas variam consoante a idade e área geográfica ou nível

1. Serviço de Cuidados Intensivos Pediátricos. Hospital Pediátrico de Coimbra. Centro Hospitalar e Universitário de Coimbra. Coimbra. Portugal.

2. Unidade de Transplantação Hepática Pediátrica e de Adultos. Hospital Pediátrico de Coimbra. Centro Hospitalar e Universitário de Coimbra. Coimbra. Portugal.

3. Unidade de Transplantação Hepática Pediátrica e de Adultos. Centro Hospitalar e Universitário de Coimbra. Coimbra, Portugal.

$\bowtie$ Autor correspondente: Carla Pinto. carla.regina.pinto@gmail.com

Recebido: 16 de Janeiro de 2015 - Aceite: 21 de Abril de 2015 | Copyright @ Ordem dos Médicos 2015 
socioeconómico da região..$^{2,5,7}$

O seu tratamento constitui um desafio para os profissionais de saúde. Combina a abordagem inicial de uma doença rapidamente progressiva com envolvimento multiorgânico e a necessidade urgente de decidir manter apenas a terapêutica médica de suporte ou avançar para um transplante hepático $(\mathrm{TRH})$ urgente..$^{8,9}$

$\mathrm{O}$ tratamento médico consiste em medidas de suporte e abordagem de complicações frequentes como distúrbios metabólicos, hidroeletrolíticos, encefalopatia hepática, hipertensão intracraniana, hemorragia, infeção e compromisso cardiovascular que podem, em última instância, evoluir para falência multiorgânica. ${ }^{1,4,5} \mathrm{O}$ TRH é o procedimento cirúrgico que, nas últimas décadas, permitiu uma significativa melhoria do seu prognóstico, aumentando a sobrevida para cerca de $60 \%$ - 85\%., $2,4,5,10-12$

Por ser rapidamente progressiva, um importante fator no prognóstico destas crianças é a sua referenciação precoce para um centro de transplantação hepática. ${ }^{11,13,14}$ A abordagem multidisciplinar e diferenciada permite uma otimização das medidas de suporte de modo a evitar o TRH ou, no caso de ser inevitável, que a criança seja submetida a esse procedimento em melhores condições. Possibilita ainda identificar situações em que o TRH está contra indicado. ${ }^{5,8,11,13,14}$ A referenciação para um centro de TRH nos primeiros seis dias de evolução de FHA associa-se a menor grau de encefalopatia e valores mais baixos de bilirubina total, assim como maior sobrevida em relação a casos com referenciação mais tardia. ${ }^{13,14}$

Em janeiro de 2008, realizou-se em Portugal uma reunião nacional multidisciplinar monotemática sobre FHA em Pediatria, que envolveu as Sociedades de Gastrenterologia, Hepatologia e Nutrição e de Cuidados Intensivos Pediátricos da Sociedade Portuguesa de Pediatria, da qual resultou um consenso de atuação reforçando a importância da sua abordagem por equipa diferenciada e a referenciação precoce para um centro com TRH. Desta forma, perante o diagnóstico de FHA em idade pediátrica, deve ser realizado um contacto com o centro de $\mathrm{TRH}$, de forma a otimizar a sua abordagem (investigação etiológica e terapêutica) e transferência precoce do doente (Pinto C. Acute liver failure: Coimbra's experience. Comunicação na Reunião Nacional Monotemática sobre Falência Hepática. Anadia, 21 de janeiro de 2008).

Este estudo teve como objetivos: apresentar a experiência e caracterizar os casos de FHA no Serviço de Cuidados Intensivos Pediátricos do único centro português com TRH pediátrico; comparar resultados em dois períodos diferentes, antes e depois da reunião de janeiro de 2008 (período $\mathrm{A}$ e $\mathrm{B}$, respetivamente).

\section{MATERIAL E MÉTODOS}

Foi realizado um estudo observacional, com colheita retrospetiva de dados, efetuado no Serviço de Cuidados Intensivos Pediátricos do Hospital Pediátrico, referência nacional para TRH pediátrico desde 1994, compreendendo um período de 20 anos e seis meses (janeiro de 1994 a junho de 2014).

Os critérios de inclusão foram idade inferior a 18 anos e FHA definida por INR $\geq 2,0$ sem resposta à administração de vitamina $\mathrm{K}$ endovenosa e evidência de necrose hepatocelular. Excluíram-se as crianças e adolescentes com doença hepática crónica.

$\mathrm{Na}$ consulta dos processos clínicos das crianças e adolescentes incluídos no estudo recolheram-se as seguintes variáveis: ano de admissão, idade, género, tempo entre o início da doença e a admissão no centro de TRH (tempo de referenciação), etiologia, apresentação clínica e laboratorial, disfunção de outros órgãos, terapêutica efetuada, índice de gravidade (pediatric risk of mortality - PRISM score) ${ }^{15}$ e mortalidade.

Foram definidos e comparados dois períodos de estudo: antes (período A) e depois (período B) da reunião monotemática de 2008. A análise estatística foi realizada com o programa Statistical Package for the Social Scien$\mathrm{ce}^{\circledR}$ versão 20 . Procedeu-se à caracterização da população por cálculo de medidas de tendência central e de dispersão para variáveis quantitativas e pela determinação de frequências absolutas e relativas para variáveis qualitativas. Para comparar variáveis nominais, foram utilizados os testes de qui-quadrado ou exato de Fisher, de acordo com as regras de Cochran. Em relação às variáveis numéricas foram efetuados os testes $t$-student ou U-Mann-Whitney após a aplicação de um teste de normalidade. Considerou-se um nível de significância de $5 \%$.

\section{RESULTADOS}

Durante o período considerado cumpriram critérios de inclusão 50 crianças/adolescentes com FHA, 28 do género feminino e 22 do masculino com uma relação 1,3:1. A idade mediana à data da referenciação foi de 24,5 (p25: 3,6; p75: $69)$ meses. Vinte e seis crianças $(52 \%)$ tinham menos de dois anos, das quais seis eram recém-nascidos.

Quarenta e seis (92\%) foram transferidos de outros hospitais ou maternidades. Vinte e um residiam na região centro, 15 foram referenciados da região sul, 11 do norte e dois das ilhas.

O tempo mediano de referenciação foi de quatro (p25: 2; p75: 14) dias.

Não foi possível identificar a etiologia da FHA em 32\% $(16 / 50)$ dos casos. A causa mais frequente na faixa etária abaixo dos dois anos foi metabólica e acima dessa idade foi infeciosa (Tabela 1).

A icterícia foi a forma de apresentação clinica mais frequente $(64 \%$; 32/50), seguida de hepatomegália $(60 \%$; $30 / 50)$. Tinham encefalopatia na admissão $56 \%$ (28/50) dos casos, sendo esta de grau igual ou superior a II em $68 \%$ (19/28). Verificou-se a presença de esplenomegália em $20 \%$ (9/45) e ascite em 20,4\% (10/49).

$\mathrm{Na}$ Tabela 2 encontram-se descritas as características laboratoriais na admissão dos doentes com FHA. A mediana do INR na admissão foi de 3,7 e 38\% (19/50) dos doentes apresentava um valor igual ou superior a 4 . Apresentavam um valor de bilirrubina total igual ou superior a 235 
Tabela 1 - Etiologia e distribuição de acordo com a faixa etária dos casos de FHA

\begin{tabular}{|c|c|c|c|}
\hline \multirow[b]{2}{*}{ Etiologia } & \multirow[b]{2}{*}{$\mathbf{n}$} & \multicolumn{2}{|c|}{ Grupo etário } \\
\hline & & $\begin{array}{l}<2 \text { anos } \\
(n=26)\end{array}$ & $\begin{array}{l}\geq 2 \text { anos } \\
(n=24)\end{array}$ \\
\hline Infeciosa & 10 & 3 & 7 \\
\hline Hepatite A & 3 & 0 & 3 \\
\hline Hepatite B & 1 & 0 & 1 \\
\hline Hepatite E & 1 & 0 & 1 \\
\hline Vírus Epstein-Barr & 1 & 1 & 0 \\
\hline Vírus Herpes-simplex 1 & 1 & 1 & 0 \\
\hline Vírus Herpes-humano 6 & 1 & 0 & 1 \\
\hline Vírus Varicela-zoster & 1 & 1 & 0 \\
\hline Leptospirose & 1 & 0 & 1 \\
\hline Metabólica & 10 & 9 & 1 \\
\hline Tirosinemia & 2 & 2 & 0 \\
\hline Défice de ОтС & 2 & 1 & 1 \\
\hline Doença mitocondrial (DGUOK) & 2 & 2 & 0 \\
\hline Galactosemia & 1 & 1 & 0 \\
\hline Frutosemia & 1 & 1 & 0 \\
\hline LCHAD & 1 & 1 & 0 \\
\hline CDG tipo $\mathrm{x}$ & 1 & 1 & 0 \\
\hline Síndrome hemofagocitico & 3 & 3 & 0 \\
\hline Doença de Wilson & 2 & 0 & 2 \\
\hline Intoxicação por cogumelos & 5 & 1 & 4 \\
\hline Imune & 2 & 1 & 1 \\
\hline Miscelânea* & 2 & 0 & 2 \\
\hline Desconhecida & 16 & 9 & 7 \\
\hline \multicolumn{4}{|c|}{$\begin{array}{l}\text { OTC: Ornitina carbamilase; DGUOK: Gene deoxiguanosina cinase; LCHAD: Long-chain 3-hydroxyacyl-CoA dehydrogenase deficiency; CDG: Defeito congénito da glicosilação. } \\
\text { * Síndrome Budd-Chiari e sindrome linfoproliferativo. }\end{array}$} \\
\hline Avaliação laboratorial na admissão & \multicolumn{3}{|c|}{$n=50$} \\
\hline INR (média, DP) & \multicolumn{3}{|c|}{$3,7 \pm 1,7$} \\
\hline Bilirrubina total (mediana, $P_{25-} P_{75}, \mu \mathrm{mol} / L$ ) & \multicolumn{3}{|c|}{$183(58-334)$} \\
\hline Glicose (mediana, $\mathbf{P}_{25-} \mathbf{P}_{75}, \mathrm{mmol} / \mathrm{L}$ ) & \multicolumn{3}{|c|}{$4,9(3,9-6,1)$} \\
\hline Amónia (mediana, $P_{25-75}, \mu \mathrm{mol} / \mathrm{L}$ ) & \multicolumn{3}{|c|}{$70(44-138)$} \\
\hline Lactato (mediana, $P_{25} P_{75}, \mathrm{mmol} / \mathrm{L}$ ) & \multicolumn{3}{|c|}{$2,8(1,8-4,6)$} \\
\hline Défice de base (média, DP) & \multicolumn{3}{|c|}{$0,017 \pm 5,8$} \\
\hline Leucócitos (mediana, $\left.P_{25-} P_{75}, \times 10^{9} / L\right)$ & \multicolumn{3}{|c|}{$9,0(6,47-11,2)$} \\
\hline Sódio (mediana, $P_{25} \cdot P_{75}, \mathrm{mmol} / L$ ) & \multicolumn{3}{|c|}{$136(132-140)$} \\
\hline Fósforo (média, DP, mmol/L) & \multicolumn{3}{|c|}{$1,2 \pm 0,4$} \\
\hline
\end{tabular}


$\mu \mathrm{mol} / \mathrm{L} 32 \%(16 / 50)$ e $48 \%$ (24/50) tinham uma contagem de leucócitos igual ou superior a $9000 / \mu \mathrm{L}$. Cinco doentes $(10 \%)$ apresentavam uma glicémia igual ou inferior a 2,8 $\mathrm{mmol} / \mathrm{L}$.

Durante o internamento em cuidados intensivos, trinta $(60 \%)$ necessitaram de ventilação mecânica invasiva e vinte e quatro (48\%) de suporte cardiovascular. Em sete (14\%) casos foram efetuadas técnicas de depuração extra-renal: hemodiafiltração venovenosa contínua em quatro, molecular adsorbents recirculation system (MARS) em dois e hemocarboperfusão num caso de intoxicação por cogumelos.

Vinte e três doentes (46\%) foram submetidos a TRH, cinco dos quais faleceram: três evoluíram para morte cerebral por hipertensão intracraniana e dois no decurso de falência multiorgânica.

Vinte e sete doentes não foram submetidos a TRH por contraindicação à sua realização (11), regeneração hepática (8) ou doenças com tratamento específico (7). Destes 27, 12 faleceram, dos quais 11 apresentavam contraindicação para TRH e um faleceu enquanto aguardava TRH, por hemorragia intracraniana.

A mortalidade global foi 34\% (Fig. 1).

A mediana do risco de mortalidade prevista pelo PRISM foi 9,1\% (p25: 3,7; p75: 27,4).

Na Fig. 2 podem visualizar-se as curvas de sobrevida livre de TRH (30\%) e pós-TRH (78\%) (curvas de Kaplan-
-Meier).

$\mathrm{Na}$ Tabela 3 descreve-se a clinica e avaliação laboratorial na admissão nos períodos $\mathrm{A}$ e $\mathrm{B}$.

A mediana do tempo de referenciação foi sete dias no período $\mathrm{A}$ e dois dias no $\mathrm{B}$ ( $p=0,006$; teste U-Mann-Whitney).

A mediana do risco de mortalidade prevista pelo PRISM foi $14,7 \%$ (p25: 4,6; p75: 40,7) no período A e 6,5\% (p25: 1,8; $p 75: 9,1)$ no B ( $p=0,019$; teste U-Mann-Whitney) (Fig. 3).

Ocorreram 13 (37\%) óbitos no período A e quatro no B $(26 \%)(p=0,474$; teste exato de Fisher).

\section{DISCUSSÃO E CONCLUSÕES}

O Serviço de Cuidados Intensivos Pediátricos onde efetuámos este trabalho é o centro de referência nacional para a FHA e o único que presta assistência a doentes em pós-operatório de TRH em idade pediátrica, desde 1994. Assim, esta é a primeira casuística de FHA nesta faixa etária publicada em Portugal. Inclui todos os casos de FHA admitidos na nossa instituição desde a implementação do programa de TRH pediátrico.

A FHA é uma patologia rara, cuja verdadeira incidência na população pediátrica é desconhecida. ${ }^{5}$ De forma similar, também em Portugal, na população em geral, não existe descrição da sua incidência. Segundo os dados aqui apresentados, nos últimos 20 anos, surgiram em média 2,5

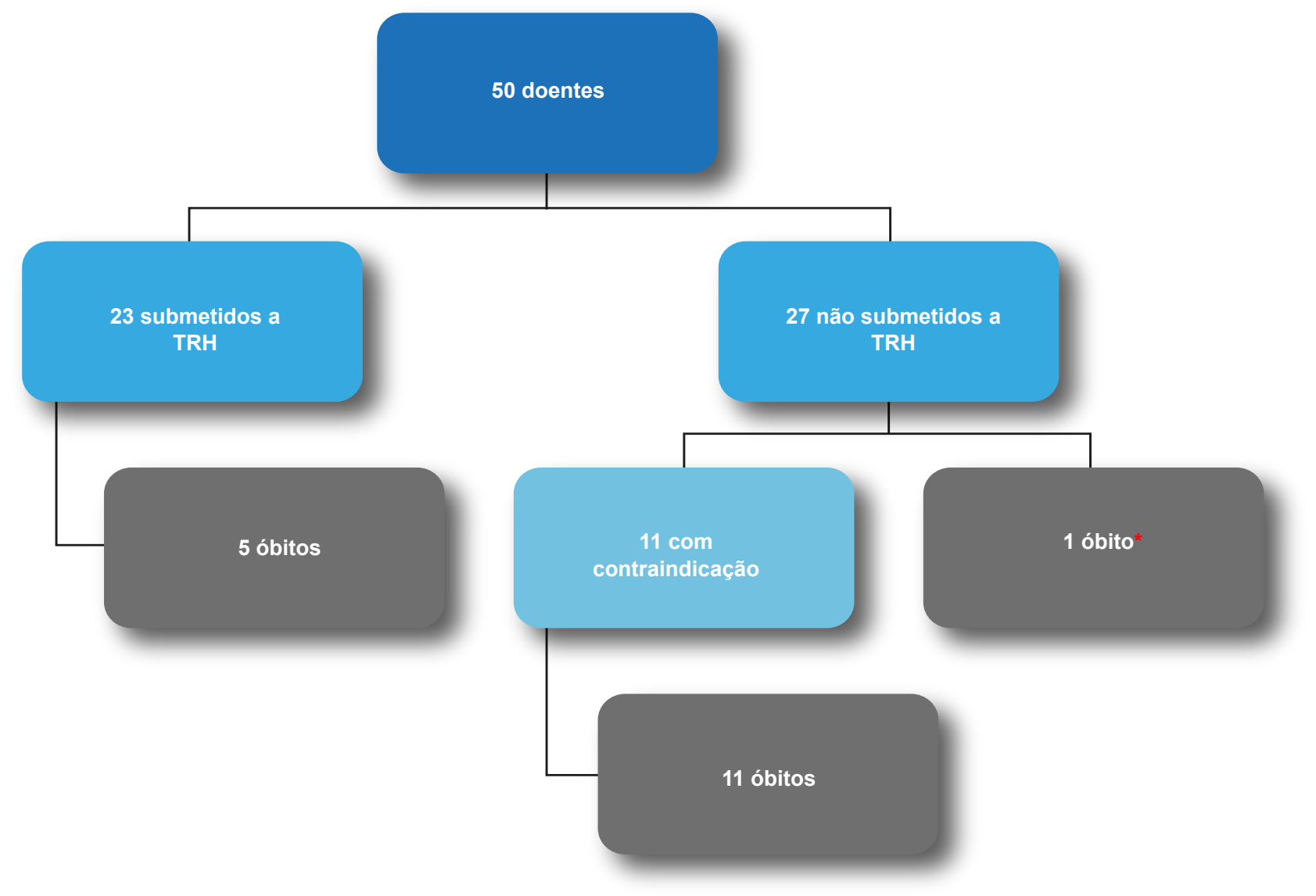

Figura 1 - Algoritmo dos óbitos nos doentes com FHA

TRH: Transplante hepático. * Em lista para TRH. 



Figura 2 - Sobrevida livre de TRH (à esquerda) e pós-TRH (à direita)

Tabela 3 - Clinica e avaliação laboratorial nos períodos A e B e respetiva comparação

\begin{tabular}{|c|c|c|c|}
\hline Características clínicas e laboratoriais na admissão & Período A $(n=35)$ & Período $B(n=15)$ & $p$ \\
\hline Idade (mediana, $\mathrm{P}_{25}-\mathrm{P}_{75}$, meses) & $15(4-67)$ & $32(1,2-90)$ & $0,589^{*}$ \\
\hline Género (Feminino, \%) & 18 & 10 & $0,423+$ \\
\hline Icterícia (\%) & 65 & 67 & $0,894 \dagger$ \\
\hline Encefalopatia (\%) & 67 & 40 & $0,082 \dagger$ \\
\hline Hepatomegália (\%) & 75 & 64 & $0,469 \neq$ \\
\hline Esplenomegália (\%) & 23 & 14 & $0,698 \neq$ \\
\hline Ascite (\%) & 21 & 20 & $1 \neq$ \\
\hline INR (média, DP) & $3,9 \pm 1,8$ & $3,2 \pm 1,5$ & $0,15 \pi$ \\
\hline Brb total (mediana, $P_{25}-P_{75}, \mu \mathrm{mol} / L$ ) & $160(58-367)$ & $185(37-254)$ & $0,488^{*}$ \\
\hline Glicose (mediana, $P_{25}-P_{75}, \mathrm{mmol} / \mathrm{L}$ ) & $4,7(3,3-5,8)$ & $5,3(4,6-7,8)$ & $0,036^{*}$ \\
\hline Amónia (mediana, $\mathrm{P}_{25}-\mathrm{P}_{75}, \mu \mathrm{mol} / \mathrm{L}$ ) & $69(46-141)$ & $70(36-92)$ & $0,747^{*}$ \\
\hline Lactato (mediana, $\mathrm{P}_{25}-\mathrm{P}_{75}, \mathrm{mmol} / \mathrm{L}$ ) & $2,8(1,8-4,1)$ & $2,7(1,5-5,8)$ & $0,911^{*}$ \\
\hline Défice de base (média, DP) & $-0,9 \pm 5,9$ & $2,5 \pm 4,9$ & $0,056 \uparrow$ \\
\hline Leucócitos (mediana, $\left.P_{25}-P_{75}, \times 10^{9} / L\right)$ & $8,7(6,1-11,2)$ & $9,1(7,6-12,2)$ & $0,616^{*}$ \\
\hline Sódio (média, DP, mmol/L) & $134 \pm 8$ & $138 \pm 4$ & $0,109 \pi$ \\
\hline Fósforo (média, DP, mmol/L) & $1,13 \pm 0,4$ & $1,25 \pm 0,3$ & $0,035 \pi$ \\
\hline
\end{tabular}

P: Percentil; INR: Índice internacional normalizado; Brb: Bilirrubina; DP: Desvio padrão. * Teste U-Mann-Whitney; †Teste qui quadrado; ¥Teste de Fisher; $\uparrow$ Teste $t$-student.

casos por ano. Ainda assim, provavelmente este valor estará subestimado uma vez que nem todos os casos ocorridos em Portugal são referenciados ao nosso centro.

A definição de FHA em idade pediátrica é distinta da do adulto, que implica a existência de encefalopatia. ${ }^{6,12} \mathrm{Na}$ criança, sobretudo nos lactentes, a encefalopatia é de difícil diagnóstico, podendo surgir tardiamente ou nunca ser clinicamente aparente. ${ }^{4,6}$ Por outro lado, numa das maiores séries sobre FHA em idade pediátrica, $20 \%$ das crianças que nunca apresentaram encefalopatia necessitaram de TRH ou faleceram. ${ }^{4}$ Desta forma, a definição mais consensual de FHA em idade pediátrica inclui a presença de coagulopatia (INR igual ou superior a 2 sem resposta à vitamina K) perante evidência de necrose hepatocelular numa 

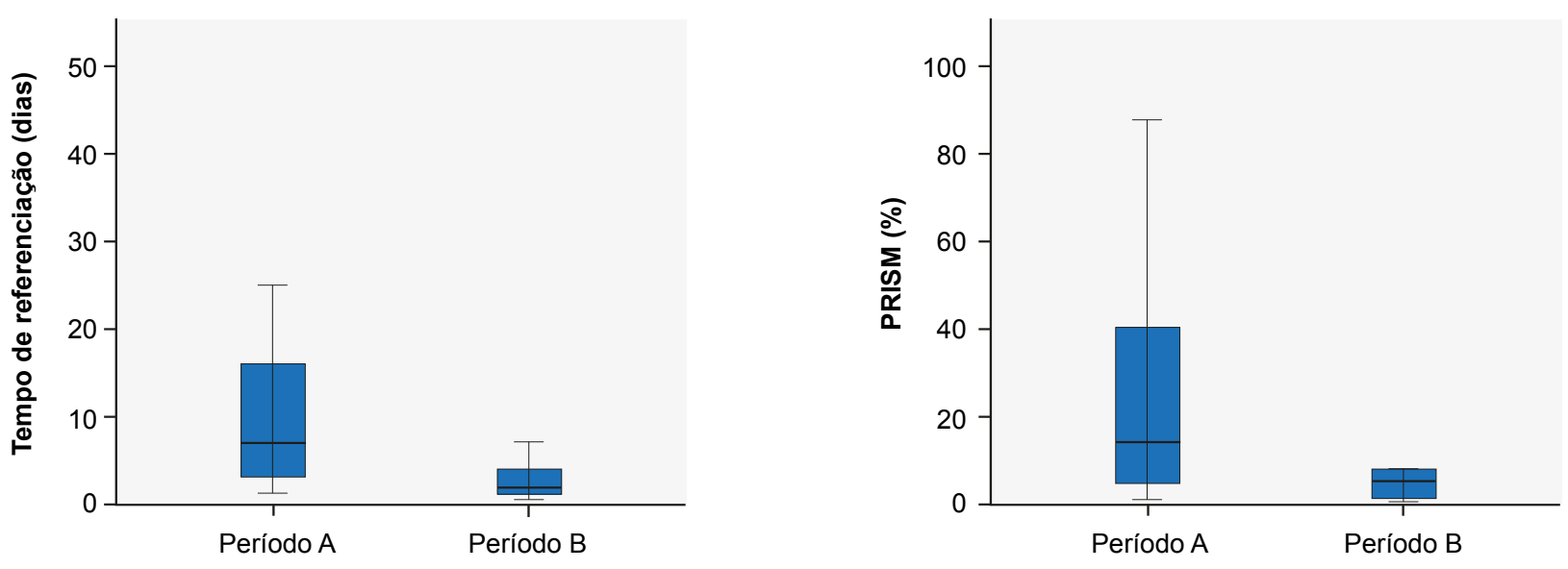

Figura 3 - Comparação entre tempo de referenciação e PRISM nos períodos A e B

PRISM: Pediatric Risk of Mortality Score

criança sem história prévia de doença hepática. ${ }^{2,4,5}$

A etiologia na criança também é diferente da do adulto e variável em função da faixa etária, área geográfica e nível socioeconómico da região. ${ }^{2,7} \mathrm{Em}$ geral, as causas infeciosas virais, são mais frequentes nos países em vias de desenvolvimento, ao passo que nos países desenvolvidos predominam as tóxicas, induzidas por fármacos. ${ }^{2,6,7} \mathrm{~A}$ intoxicação por paracetamol constitui a causa mais frequente de FHA acima dos três anos tanto nos Estados Unidos da América como na Europa. ${ }^{6,12}$ Contrariamente, no nosso estudo não houve nenhum caso de FHA por intoxicação com paracetamol. Na nossa série, a causa mais frequente na faixa etária acima dos dois anos foi infeciosa (29\%), com predomínio da hepatite $A(n=3)$. Portugal é, atualmente, considerado um país com baixos níveis de infeção por hepatite A devido a uma melhoria das condições sanitárias e de higine. ${ }^{16}$ Um estudo não publicado realizado em 2003 na nossa instituição, incluindo 255 jovens com idade entre um e 14 anos da região centro, mostrou uma prevalência global de anticorpos anti vírus da hepatite A de 9,4\% (IC $95 \% 5,8 \%$ - 13\%). A maioria dos casos de doença surge de forma episódica e associados a populações migratórias. ${ }^{16}$

Nas idades mais jovens, as doenças metabólicas contribuem para a maioria dos casos de FHA, estando descritos valores na ordem dos $10 \% .{ }^{6}$ No nosso estudo estas foram ainda mais frequentes, constituindo $35 \%$ dos casos abaixo dos dois anos. A hepatite autoimune contribuiu para $4 \%$ dos casos, valor semelhante ao de outras séries. ${ }^{6}$ É ainda de salientar a intoxicação por cogumelos, causa importante de FHA na Europa ocidental, nomeadamente em Portugal. ${ }^{4}$ No nosso estudo, ocorreu em $10 \%$ dos casos, sendo descrita uma prevalência de $5 \%$ noutro estudo realizado num Serviço de Cuidados Intensivos de adultos da região centro de Portugal. ${ }^{17}$ A FHA de etiologia indeterminada ocorreu em $32 \%$ dos casos, valor inferior ao descrito na literatura $(46 \%$ a $49 \%) \cdot{ }^{6,8}$ A investigação etiológica deve ser encorajada, uma vez que possibilita a identificação de potenciais causas tratáveis e, por outro lado, de doenças com envolvimento multissistémico que possam constituir contraindicação ao
TRH. ${ }^{5}$ Tal como descrito noutros estudos de centros com maior experiência e capacidade de investigação, a nossa menor percentagem de causas desconhecidas pode ser explicada pelo fato de sermos centro de referência. ${ }^{18}$ Outro fator também descrito associado a elevada percentagem de causas indeterminadas é a gravidade clínica da FHA com rápida evolução para necessidade de TRH ou óbito, impedindo uma atempada investigação etiológica. ${ }^{5}$

Relativamente à clinica de apresentação, a icterícia foi a manifestação mais frequente $(64 \%)$ à semelhança de outros estudos. ${ }^{3} \mathrm{O}$ segundo achado clinico mais frequente foi a hepatomegália, presente em $60 \%$ dos casos. A encefalopatia foi diagnosticada em $56 \%$ dos doentes, valor sobreponível ao de outros estudos, que descrevem valores entre $50 \%$ e $54 \%$. 6,8

A mediana do risco de mortalidade previsto pelo PRISM foi $9,1 \%$, o que, comparativamente à mortalidade real (34\%), revela uma provável inadequação deste índice como fator de prognóstico na FHA, fato já descrito por alguns autores. ${ }^{5,19} \mathrm{O}$ PRISM é um índice de prognóstico calculado com base em parâmetros obtidos nas primeiras 24 horas após admissão em cuidados intensivos pediátricos. ${ }^{15}$ Tendo em conta que a FHAé uma doença rapidamente progressiva e que, idealmente os doentes são admitidos ainda numa fase precoce, provavelmente este índice não será apropriado para avaliação do prognóstico, mas poderá ser útil na determinação da gravidade dos doentes na admissão.

Outro índice de prognóstico utilizado em cuidados intensivos pediátricos - pediatric index of mortality (PIM), também tem sido descrito como inadequado para este grupo. ${ }^{20}$

A FHA apresenta habitualmente uma evolução rapidamente progressiva pelo que é fundamental identificar os doentes que podem beneficiar de $\mathrm{TRH}$, a terapêutica mais eficaz, definitiva e associada a melhor sobrevida. ${ }^{12} \mathrm{Na}$ era pré-TRH, a mortalidade associada a esta patologia rondava os $70 \%-75 \%$. $^{3,12,21}$ Atualmente, a FHA contribui para cerca de $15 \%$ dos $\mathrm{TRH}$ pediátricos, com uma significativa 
melhoria da sobrevida. ${ }^{10,12}$ Há, no entanto, a salientar que o TRH, em caso de FHA, está associado a pior sobrevida quando comparado com as suas outras indicações (sobrevida a um ano de $74 \%$ na FHA vs $88,2 \%$ se TRH por outra causa). ${ }^{4,21}$ Neste estudo, a sobrevida pós-TRH (78\%) foi sobreponível à de outros centros europeus. ${ }^{4,5,11} \mathrm{~A}$ sua diferença relativamente à sobrevida livre de $\mathrm{TRH}(30 \%)$ vem reforçar que o TRH é de fato a terapêutica mais eficaz e que mais influencia a sobrevida destes doentes.

A existência de um índice de prognóstico adequado seria um importante auxiliar na abordagem dos doentes com FHA, permitindo uma utilização judiciosa de órgãos disponíveis e evitando o TRH e suas consequências em doentes com grande probabilidade de regeneração. ${ }^{21}$ Existem vários modelos de prognóstico utilizados em adultos. O mais amplamente utilizado, King's College Hospital Criteria, não é aplicável à população pediátrica nos casos cuja etiologia não é devida à intoxicação por paracetamol. ${ }^{21}$ Assim, não existem, até ao momento, índices de prognóstico nem critérios de indicação para TRH validados para a idade pediátrica. ${ }^{4,12}$ No entanto, consideram-se fatores de mau prognóstico, com base num estudo realizado num pequeno número de doentes na era pré-transplante no King's College Hospital, os seguintes: INR $\geq 4,0$; bilirrubina total $\geq 235$ $\mu \mathrm{mol} / \mathrm{L}$; idade inferior a dois anos e contagem de leucócitos $\geq 9 \times 10^{9} /$ L. $^{2,11}$ Existe também um score que parece promissor (LIU score) e que inclui uma fórmula matemática que utiliza os valores laboratoriais da bilirrubina sérica, tempo de protrombina (ou INR) e amónia. No entanto, não foi ainda validado para poder ser amplamente utilizado. ${ }^{22}$

A decisão de transplantar é baseada habitualmente na clinica, avaliação analítica e evolução do doente, sendo fatores de extrema importância a encefalopatia e a elevação progressiva do INR. ${ }^{4}$ Outros critérios são a presença de encefalopatia de grau igual ou superior a 2 associada a uma atividade do fator $V$ inferior a $20 \%$, fibrinogénio inferior a 1 $\mathrm{g} / \mathrm{L}$, hiperbilirrubinémia superior a $400 \mu \mathrm{mol} / \mathrm{L}$, hiperamoniémia superior a $150 \mathrm{mmol} / \mathrm{L}$, entre outros. ${ }^{5,23}$

Um número significativo $(54 \%, 27 / 50)$ de crianças e adolescentes não foi submetido a TRH por contraindicação à sua realização (11), regeneração hepática (8) ou doenças com tratamento específico (7). O único caso não submetido a TRH e que estava em lista faleceu por hemorragia intracraniana.

A FHA pode ser rapidamente progressiva com agravamento clinico em poucas horas, pelo que o contacto precoce com os centros de TRH é fundamental. ${ }^{2,8,11,12}$ Mesmo os doentes sem encefalopatia ou com encefalopatia grau I ou II podem agravar de forma muito rápida, e por isso é necessário reconhecer precocemente a FHA. ${ }^{12} \mathrm{~A}$ mediana do tempo de referenciação deste estudo foi globalmente de 4 dias, inferior ao descrito (12 - 14 dias). ${ }^{8}$ Após a reunião nacional de 2008 , houve uma redução estatisticamente significativa deste tempo no período B ( 2 vs 7 dias no período A). Por outro lado, a mediana do risco de mortalidade previsto pelo PRISM também diminuiu significativamente após $2008(14,7 \%$ vs $6,5 \%)$ sinal de que provavelmente os doentes foram admitidos no Serviço de Cuidados Intensivos apresentando um estado clinico menos grave, o que é também sugerido pela tendência na redução no valor de INR $(3,9$ vs 3,2) e encefalopatia ( $67 \%$ vs $40 \%)$. Apesar de existir uma tendência na redução da mortalidade após 2008 (37\% vs 26\%), esta não teve ainda significado estatístico, provavelmente devido ao pequeno número de doentes do estudo sobretudo após 2008.

A importância da referenciação precoce para centros terciários tem sido descrita por alguns autores. Parece estar associada a menor número de indicadores de mau prognóstico, nomeadamente presença de encefalopatia, e, por isso, diretamente relacionada com uma melhoria na sobrevida. ${ }^{13,14}$

Este estudo é limitado por ser retrospetivo e ter um pequeno número de casos, apesar de ser de um centro de referência, sugerindo-se a realização de estudos multicêntricos internacionais prospetivos que possam responder a questões fundamentais como fatores de prognóstico e critérios para transplante hepático em pediatria.

Em conclusão, a FHA é uma patologia associada a elevada morbilidade e mortalidade, apesar da melhoria da sobrevida com o TRH. Tendo em conta a sua rápida evolução, é fundamental a abordagem multidisciplinar, diferenciada, estruturada e estandardizada, por uma equipa experiente. Após 2008 o tempo de referenciação e a gravidade dos casos na admissão reduziram, mas ainda sem tradução significativa na mortalidade.

Assim, a referenciação precoce é fundamental, bem como estabelecer uma monitorização prospetiva dos nossos resultados, de forma a demonstrar no futuro o impacto que esta terá na mortalidade, com um maior número de casos.

\section{PROTECÇÃO DE PESSOAS E ANIMAIS}

Os autores declaram que os procedimentos seguidos estavam de acordo com os regulamentos estabelecidos pelos responsáveis da Comissão de Investigação Clínica e Ética e de acordo com a Declaração de Helsínquia da Associação Médica Mundial.

\section{CONFIDENCIALIDADE DOS DADOS}

Os autores declaram ter seguido os protocolos do seu centro de trabalho acerca da publicação de dados.

\section{CONFLITOS DE INTERESSE}

Os autores declaram não terem qualquer conflito de interesse relativamente ao presente artigo.

\section{FONTES DE FINANCIAMENTO}

Os autores declaram não ter recebido subsídios ou bolsas para a elaboração do artigo. 


\section{REFERÊNCIAS}

1. Bhatia V, Lodha R. Intensive care management of children with acute liver failure. Indian J Pediatr. 2010;77:1288-95.

2. Dhawan A. Acute liver failure in children and adolescents. Clin Res Hepatol Gastroenterol. 2012;36:278-83.

3. Durand P, Debray D, Mandel R, Baujard C, Branchereau S, Gauthier F, et al. Acute liver failure in infancy: a 14-year experience of a pediatric liver transplantation center. J Pediatr. 2001;139:871-6.

4. Squires $\mathrm{RH}$. Acute liver failure in children. Semin Liver Dis. 2008;28:153-66

5. Devictor D, Tissieres P, Durand P, Chevret L, Debray D. Acute liver failure in neonates, infants and children. Expert Rev Gastroenterol Hepatol. 2011;5:717-29.

6. Squires RH, Shneider BL, Bucuvalas J, Alonso E, Sokol RJ, Narkewicz $M R$, et al. Acute liver failure in children: the first 348 patients in the pediatric acute liver failure study group. J Pediatr. 2006;148:652-8.

7. Kayaalp C, Ersan V, Yilmaz S. Acute liver failure in Turkey: a systematic review. Turk J Gastroenterol. 2014;25:35-40.

8. Strauss A, Grabhorn E, Sornsakrin M, Briem-Richter A, Fischer L, Nashan B, et al. Liver transplantation for fulminant hepatic failure in infancy: a single center experience. Pediatr Transplant. 2009;13:83842.

9. Bernal W, Auzinger G, Sizer E, Wendon J. Intensive care management of acute liver failure. Semin Liver Dis. 2008;28:188-200.

10. Heffron TG, Pillen T, Smallwood G, Rodriguez J, Sekar S, Henry S, et al. Pediatric liver transplant for acute liver failure at a single center: a 10-yr experience. Pediatr Transplant. 2010;14:228-32.

11. Nadalin S, Heuer M, Wallot M, Auth M, Schaffer R, Sotiropoulos GC, et al. Paediatric acute liver failure and transplantation: the university of Essen experience. Transpl Int. 2007;20:519-27.

12. Lee WM, Larson AM, Stravitz RT. AASLD Position Paper: The management of acute liver failure: Update 2011. [consultado em 2014 Nov 15]; Acessível em: http://www.aasld.org/practiceguidelines/ Documents/AcuteLiverFailureUpdate2011.pdf.

13. Sturm E, Lexmond WS, Verkade HJ. Pediatric acute liver failure: variations in referral timing are associated with disease subtypes. Eur J Pediatr. 2015;174:169-75.

14. Kremers L, Verkade HJ, Sturm E. Acute liver failure in children: relation between duration of pre-tertiary care, symptoms and outcome. $J$ Pediatr Gastroenterol Nutr. 2006;42:E74.

15. Pollack MM, Ruttimann UE, Getson PR. Pediatric risk of mortality (PRISM) score. Crit Care Med. 1988;16:1110-6.

16. Antunes $\mathrm{H}$, Macedo $\mathrm{M}$, Estrada $\mathrm{A}$. Prevalência do vírus da hepatite $A$ primeiros resultados de baixa endemicidade em Portugal. Acta Med Port. 2004;17:219-24.

17. Areia M, Romãozinho JM, Ferreira M, Amaro P, Leitão MC. Fulminant hepatic failure: a Portuguese experience. Eur J Gastroenterol Hepatol. 2007; 19:665-9.

18. Narkewicz MR, Olio DD, Karpen SJ, Murray KF, Schwarz K, Yazig $\mathrm{N}$, et al. Pattern of diagnostic evaluation for the causes of pediatric acute liver failure: an opportunity for quality improvement. J Pediatr. 2009;155:801-6.

19. Tissières $P$, Chevret $L$, Devictor $D$. The pediatric risk of mortality score in infants and children with fulminant liver failure. Pediatr Transplant. 2003;7:64-8.

20. Matthews CE, Goonasekera C, Dhawan A, Deep A. Validity of pediatric index of mortality 2 (PIM2) score in pediatric acute liver failure. Critical Care. 2014;18:665.

21. Sundaram V, Shneider BL, Dhawan A, Ng VL, Im K, Belle S, et al King's College Hospital Criteria for non-acetaminophen induced acute liver failure in an international cohort of children. J Pediatr. 2013;162:319-23.

22. Lu BR, Zhang S, Narkewicz MR, Belle SH, Squires RH, Sokol RJ Evaluation of the liver injury unit scoring system to predict survival in a multinational study of pediatric acute liver failure. J Pediatr. 2013;162:1010-6.

23. Lu BR, Gralla J, Liu E, Dobyns EL, Narkewicz MR, Sokol RJ. Evaluation of a scoring system for assessing prognosis in pediatric acute liver failure. Clin Gastroenterol Hepatol. 2008;6:1140-5. 
Mónica JERÓNIMO, Rita MOINHO, Carla PINTO, Leonor CARVALHO, Isabel GONÇALVES, Emanuel FURTADO, José FARELA NEVES

\section{A Importância da Referenciação Precoce na Falência Hepática Aguda Pediátrica}

Acta Med Port 2015:28:559-566

Publicado pela Acta Médica Portuguesa, a Revista Científica da Ordem dos Médicos

Av. Almirante Gago Coutinho, 151

1749-084 Lisboa, Portugal.

Tel: +351218428215

E-mail: submissao@actamedicaportuguesa.com

www.actamedicaportuguesa.com

ISSN:0870-399X | e-ISSN: 1646-0758

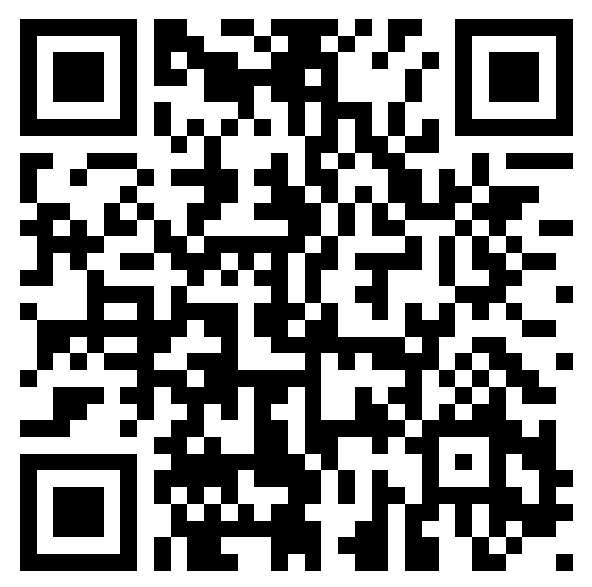

